This item was submitted to Loughborough's Research Repository by the author.

Items in Figshare are protected by copyright, with all rights reserved, unless otherwise indicated.

\title{
Large eddy simulation of a compressor cascade and the influence of spanwise domain
}

\section{PLEASE CITE THE PUBLISHED VERSION}

http://dx.doi.org/10.1177/0957650911409318

\section{PUBLISHER}

(C) SAGE Publications on behalf of the Institution of Mechanical Engineers

VERSION

VoR (Version of Record)

\section{LICENCE}

CC BY-NC-ND 4.0

\section{REPOSITORY RECORD}

McMullan, W.A., and Gary J. Page. 2012. "Large Eddy Simulation of a Compressor Cascade and the Influence of Spanwise Domain". figshare. https://hdl.handle.net/2134/9496. 
This item was submitted to Loughborough's Institutional Repository (https://dspace.lboro.ac.uk/) by the author and is made available under the following Creative Commons Licence conditions.

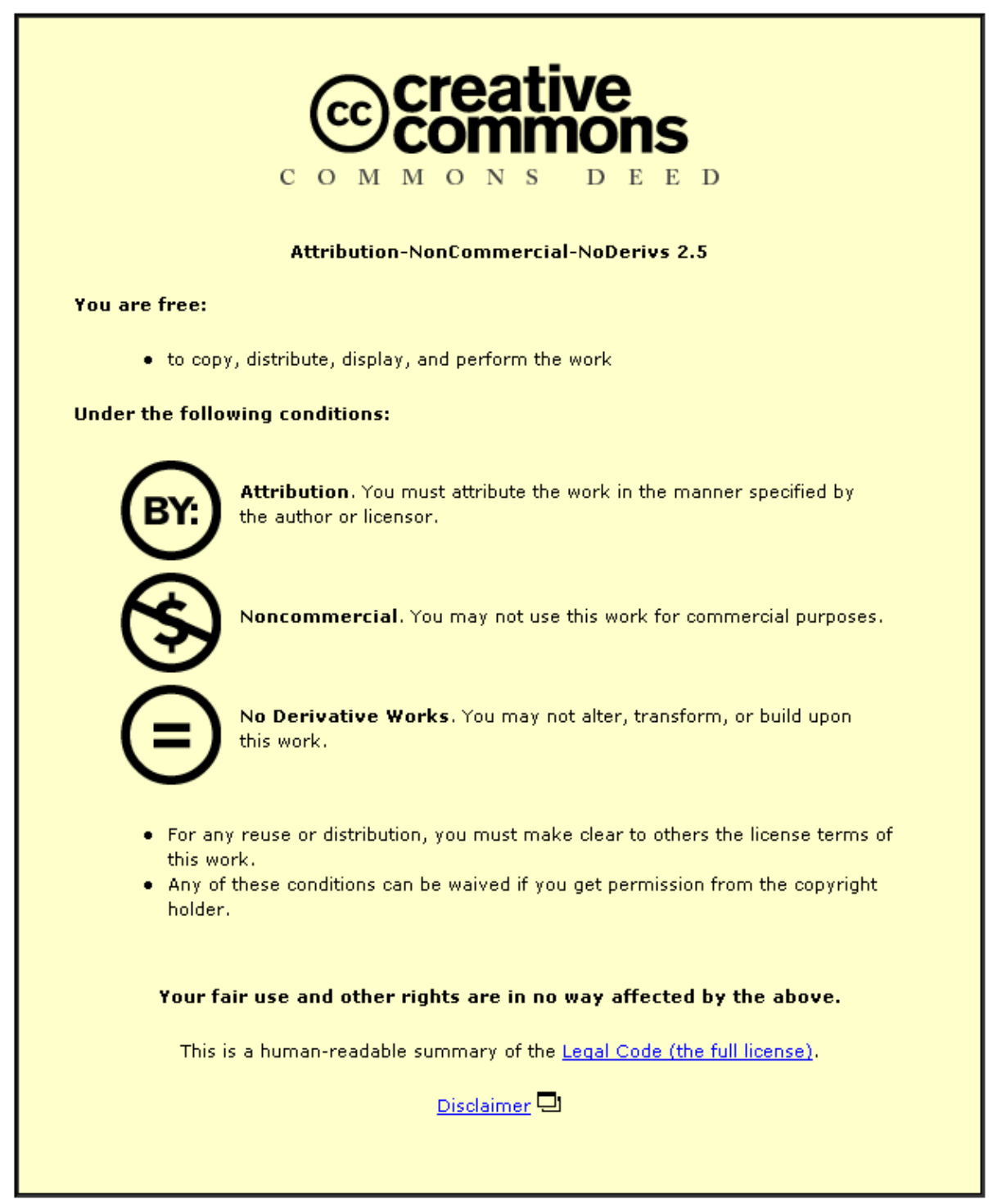

For the full text of this licence, please go to: http://creativecommons.org/licenses/by-nc-nd/2.5/ 


\title{
Large eddy simulation of a compressor cascade and the influence of spanwise domain
}

\author{
W A McMullan and G J Page* \\ Department of Aeronautical and Automotive Engineering, Loughborough University, Loughborough, UK
}

The manuscript was received on 24 November 2010 and was accepted after revision for publication on 13 April 2011.

DOI: $10.1177 / 0957650911409318$

\begin{abstract}
A controlled diffusion compressor cascade is studied using large eddy simulation (LES). The aim of this study is to assess the capability of LES to be used in an industrial context. The Reynolds number is approximately 700000 based on chord length and inlet velocity. A 'thinslice' representation of the cascade is used as the reference grid, and the influence of a narrow span is studied by comparison simulations with a domain that has a span five times larger than the thin-slice grid. While the instantaneous flow-fields of the thin-slice and wide-domain simulations are qualitatively similar, the thin-slice simulations suffer from flow confinement problems caused by the imposition of the narrow span. The non-unity axial velocity density ratio of the flow enforces the use of inviscid wall spanwise boundaries, which have a parasitic influence on the development of the flow in the thin-slice simulations. The resultant data obtained from the thinslice simulations are therefore compromised and the computed loss estimation is considered unreliable. However, when comparing mean quantities such as surface pressure and boundary layer growth the narrow does give reasonable predictions. While the inviscid spanwise walls also affect the flow near the boundaries in the wide domain simulations, there is sufficient region of span from which reliable flow data and loss estimations can be obtained. For blade flows at off-design conditions, a span of 20 per cent of the blade chord is sufficient to give good agreement with experimental data. This incurs a computational cost that may be too high to incorporate parametric LES studies into the design cycle of turbomachinery components with current computers.
\end{abstract}

\section{INTRODUCTION}

In an industrial environment, Reynolds-averaged Navier-Stokes (RANS) models have been the standard simulation tool in a wide variety of flow configurations for a number of years. The relatively coarse grids required by RANS and its subsequently lowcomputational cost allows flow solutions to be obtained rapidly, and many design parameters can be considered in a short period of time. This ensemble-averaging approach to the solution of the governing equations does, however, come at some expense

* Corresponding author: Department of Aeronautical and Automotive Engineering, Loughborough University, Ashby Road, Leicestershire LE11 3TU, UK.

email: G.J.Page@lboro.ac.uk as no time-dependent flow data are available that could potentially benefit a design process. Modern supercomputing power has now brought fully timedependent simulation within reach of the industrial design process. As the computational expense of direct numerical simulation (DNS) limits its applicability to flows of moderate Reynolds numbers, it is expected that large eddy simulation (LES) will become increasingly relevant to industrial applications in the foreseeable future.

Published research into the use of time-dependent simulation in turbomachinery flows has become more apparent in the past few years. The majority of this study have focused on the simulation of turbine blades in linear cascades. While computationally expensive, DNS has been performed for such turbine cascades at transitional Reynolds numbers [1]. LES of 
turbine cascades have been performed at conditions that match reference DNS data [2], and it was found that the LES predicted a transition on the suction side of the blade that was delayed by some 10 percent when compared to the DNS. This was attributed to the coarse grid used in the simulation and highlights the resolution requirements for wallbounded simulations. In all of the above simulations, the incoming wakes were included in the simulations through the use of precursor calculations [3]. The effect of small-scale turbulence embedded within the wakes was studied by Wissink et al. [4], who concluded that while the large-scale motion of the wake triggers the Kelvin-Helmholtz $(\mathrm{K}-\mathrm{H})$ instability on the suction side of the blade, the small-scale disturbances embedded into the wake flow seed the transition to turbulence in the shear layer flow. Matsuura and Kato [5] used LES to predict transitional flow in a turbine cascade and found that the dominant unsteady behaviour of the transitional boundary layers depends upon the presence of simulated freestream turbulence.

There have been surprisingly few studies of flows relevant to compressors using LES. You et al. [6] used LES to examine loss mechanisms in rotor tipclearance flows [7], including the effect of varying tip-gap size [8]. This study also acted as a catalyst to improve RANS modelling for turbomachinery with a second-moment closure [9]. Of particular relevance to this study are the DNS of a linear compressor cascade at a moderate Reynolds number by Zaki et al. [10]. A precursor DNS of the shedding from a cylinder was used to generate the periodically passing wake. This caused the suction-side flow to separate and rollup into discrete $\mathrm{K}-\mathrm{H}$ vortices, while on the pressure side the flow underwent a bypass transition due to the passing of the wake.

Of particular interest in this study is the flow in axial compressors. The idealized controlled diffusion cascade of Sanger and Shreeve [11] has been studied extensively, yielding a significant amount of experimental data over a wide range of flow inlet angles [12-16]. This large dataset has prompted the cascade to be the subject of several numerical studies of the flow $[\mathbf{1 4}, \mathbf{1 7 - 2 0}]$ with each study showing deficiencies in the prediction of the loss of the blade over a range of flow inlet angles.

The aim of this present study is to evaluate the ability of LES to predict loss coefficient prediction of the cascade of Sanger and Shreeve over a wide variety of flow inlet angles. Loss coefficient is a key parameter used to evaluate compressor designs and its accurate prediction for a range of design points is essential to allow the design of more efficient compressor systems. The idealized cascade of Sanger and Shreeve is a good test case for LES as its relatively simple configuration removes the geometrical difficulties encountered when simulating flows in realistic configurations, such as shroud- and tip-gap effects. As common practice in industry is to represent idealized blades using a thin-slice streamtube representation of the blade, a domain that has a spanwise extent of $L_{Z}=0.04 c$ will be used to test the performance of LES on such domains. The effect of imposing a narrow spanwise domain on the time-dependent simulation will be assessed through comparison with simulations that have been performed on a domain that has a spanwise domain of $L_{Z}=0.2 c$. This study is an extension of previous studies by the current authors $[\mathbf{1 9}, \mathbf{2 0}]$, where simulations were performed over a wide range of flow inlet angles on grids that had a large spanwise extent. The objective of this study is to assess the viability of LES using narrow span simulations, which are computationally viable in an industrial context, and in particular the initial assessment of loss prediction at off-design.

\section{METHODOLOGY}

Although the simple cascade geometry considered in this study is easily meshed using a multiblock hexahedral mesh, real gas turbine compressors contain geometrical features, such as cavities, which are more easily meshed using a general unstructured approach. This study uses a solver with an edgebased data structure that can transparently handle mixed element meshes (tetrahedra, prisms, pyramids, and hexahedra) [21] so that the outcomes of this study can be easily transferred to realistic geometry cases.

As compared to the baseline RANS solver, the spatial and temporal discretizations have been improved so as to avoid excessive dissipation of resolved eddies, and subgrid scale models incorporated. The important features are summarized below, and further details of the discretization and testing on some simplified flow problems can be found in Tristanto et al. [22]. The method has also been validated on multiple impinging jet problems relevant to vertical landing aircraft $[23,24]$.

\subsection{Governing equations}

The time-dependent Navier-Stokes equations for compressible flow, with spatially filtered Favreaveraged variables are

$$
\frac{\partial}{\partial t} \int_{\Gamma} Q \mathrm{~d} V+\int_{\partial \Gamma} F(Q) \cdot n \mathrm{~d} S+\int_{\partial \Gamma} G(Q) \cdot n \mathrm{~d} S=0
$$


where

$$
Q=\left[\begin{array}{c}
\bar{\rho} \\
\bar{\rho} \widetilde{u} \\
\bar{\rho} \widetilde{v} \\
\bar{\rho} \widetilde{w} \\
\widetilde{E}
\end{array}\right], F(Q) \cdot n=\left[\begin{array}{c}
\bar{\rho} \widetilde{U}_{n} \\
\bar{\rho} \widetilde{U}_{n} \widetilde{u}+\hat{n}_{x} \bar{p} \\
\bar{\rho} \widetilde{U}_{n} \widetilde{v}+\hat{n}_{y} \bar{p} \\
\bar{\rho} \widetilde{U}_{n} \widetilde{w}+\hat{n}_{z} \bar{p} \\
\widetilde{U}_{n}(\widetilde{E}+\bar{p})
\end{array}\right]
$$

and $G(Q)$ contains viscous and conduction flux terms. The notation ${ }^{-}$is for unweighted-filtered variables and $\sim$ density-weighted filtered variables.

\subsection{Discretization}

The domain is meshed using tetrahedra, hexahedra, pyramids, and prisms. This is then used to create the median dual control volumes that surround each node of the mesh.

The fluxes through the median dual control volume faces are accumulated by looping over all the edges connecting the nodes. For edge $i j$ connecting node $i$ to $j$ a second-order central difference scheme with added smoothing is used [25]

$$
F_{i j}=\frac{1}{2}\left[F\left(Q_{i}\right)+F\left(Q_{j}\right)-\left|A_{i j}\right| \varepsilon\left(L_{j}^{l p}(Q)-L_{i}^{l p}(Q)\right)\right]
$$

The smoothing is based upon the difference of the pseudo-Laplacian at the nodes [21] and so is a fourthorder term. The parameter $\varepsilon$ controls the level of smoothing.

For LES, it is important that the added smoothing term should be small so as to avoid unphysical dissipation of the resolved eddies, while still retaining a stable solution. Previous study by Ciardi et al. [26] on an earlier version of the methodology experimented with a 'wiggle detector' to automatically control the smoothing, with testing on the decay of isotropic turbulence and a fully developed channel flow. Similarly, Tristanto et al. [22] used the Ducros vorticity and divergence monitor [27] to control smoothing with results shown for a fully developed pipe flow and a Mach 0.9 free jet. For comparison, the cases were also computed with another unstructured method using Roe flux difference splitting and linear-reconstruction with a least-squares approach. Careful examination of these results showed equivalent solutions could be achieved using simple fixed smoothing coefficient $\varepsilon=0.2$ and this has been used throughout this study. This smoothing term is appropriate for a density-based solver, but close to the wall where the local Mach number reduces, the formulation can lead to larger numerical smoothing than an incompressible pressure-based method. As will be seen in the solutions presented, near wall structures are well resolved in this study, but this is an area that may require further research.

Temporal discretization uses a standard thirdorder accurate, three-stage Runge-Kutta algorithm.

\subsection{Sub grid scale model}

The Smagorinsky sub grid scale (SGS) model [28] defines the subgrid scale viscosity as

$$
\mu_{\text {sgs }}=\rho l_{\text {smag }}^{2} \sqrt{2 S_{i j} S_{i j}}
$$

where the Smagorinsky length scale is

$$
l_{\text {smag }}=C_{s} \Delta
$$

the strain rate is

$$
S_{i j}=\frac{1}{2}\left(\frac{\partial \tilde{u}_{i}}{\partial x_{j}}+\frac{\partial \tilde{u}_{j}}{\partial x_{i}}\right)
$$

and the filter width $\Delta$ is calculated from the cube root of the local finite volume control volume and $C_{s}$ is a model constant that is problem dependent.

In practical engineering calculations, hexahedral meshes resolve the gradients in the boundary layer by decreasing spacing normal to the wall, and this results in moderate- to high-aspect ratios of the control volumes close to the wall. The consequence is that the filter width, based on the cube root of the volume, stays relatively large and unrealistically high-values of $\mu_{\text {sgs }}$ are found close to the wall. This could be solved by significantly reducing the spacing in the transverse and longitudinal directions so as to achieve element aspect ratios of order unity, but there is a limit as to how fine the mesh can be made without the number of elements becoming impractical for calculation. Here, an alternative approach is taken to avoid the incorrect length scale.

In the inner region of the Baldwin-Lomax or Cebeci-Smith mixing length RANS model, the turbulent viscosity takes a similar mathematical form to the SGS viscosity of the Smagorinsky model

$$
\left(\mu_{t}\right)_{i}=\bar{\rho} l_{b l}^{2}|\bar{\omega}|
$$

where the mixing length is in this case

$$
l_{b l}=\kappa y\left[1-\exp \left(\frac{y^{+}}{A^{+}}\right)\right]
$$

As the Smagorinsky SGS model only accounts for the modelled part of the total stress, this should be smaller than the total Reynolds stress predicted by the RANS mixing length model. Hence, this can be used to restrict the length scale in the Smagorinsky model

$$
l_{\text {smag }}=\min \left(C_{s} \Delta, \kappa y\right)
$$


This SGS model length scale modification can be interpreted as using a RANS- type model for the inner region, and is similar to the detached eddy simulation (DES) approach in that a length scale is defined as the minimum of the RANS model length scale and a SGS length scale based upon the local element size. Unlike DES, which aims to use the RANS model for the whole of the boundary layer, the objective here is to use the length scale restriction only in the inner region of the boundary layer in order to improve the prediction of the wall shear stress.

For the calculations presented here, the SGS model length scale modification was typically active for a layer of only two to five elements away from the wall with the last outer element being at a position with a non-dimensional wall distance $y^{+}$less than seven. As mentioned previously, the smoothing in a density-based solver is more prone to being large in the low-speed region near the wall, and it may be important in this type of methodology to ensure that the behaviour of the SGS model does not exacerbate this.

\subsection{Parallel implementation}

To compute LES in a reasonable elapsed time, and so be viable in an industrial design process, requires efficient usage of massively parallel computers.

While structured multiblock computational fluid dynamics (CFD) codes are relatively straightforward to implement in parallel on distributed memory machines using domain decomposition by block, the unstructured solver requires the grid to be partitioned at run-time. The unstructured solver uses the OPLUS library [29] with message passing subsequently implemented using the message passing interface. The partitioning is itself carried out in parallel using the ParMetis library. The parallel implementation has been tuned for large-scale problems and near linear speed-up is demonstrated up to 1024 processors on an IBM Power5 system [30]. Calculations presented here have been run on a Cray XT-4 system using up to 1024 processor cores.

\section{CONFIGURATION}

The experiment simulated in this research is the controlled diffusion cascade at the Naval Postgraduate School in Monterey, California. This has been studied extensively and a large amount of data from the rig is available in the open literature [11-16]. The blade chord length $c$ is $0.1273 \mathrm{~m}$ giving Reynolds numbers based on inlet velocity of $600000-800000$. The experimental test cases considered in this study are defined in Table 1. The experiments were carried out for a variety of inlet angles $\beta$ with $37-40^{\circ}$ being considered as 'on-design', while $24-32^{\circ}$ is 'off-design' with a negative incidence and $43-46^{\circ}$ is 'off-design' with a positive incidence. The off-design cases are particularly important tests for LES as these lead to larger losses and are more difficult to compute with RANS CFD.

Two computational grids, differing in their spanwise extent are used in this study. Each grid has the same axial and transverse extent, with the distribution of grid points in these directions also identical. The number of axial nodes is set to 370 on each surface of the blade, with nodes clustered towards the leading and trailing edges. The grid normal to the blade surface has a first-cell height that gives a nondimensional wall distance $y^{+} \approx 4$ at the leading edge, and $y^{+} \approx 2.2$ at the trailing edge. The minimum $y^{+} \approx 1.2$ occurs at approximately 60 per cent chord on the pressure-side surface. The streamwise spacing varies between $x^{+} \approx 15$ at the leading and trailing edges to $x^{+} \approx 50$ at mid-chord.

The first grid (LES-N) is a 'thin-slice' representation of the blade geometry, with the span of the blade is set to $L_{z}=0.04 c$. Eighty nodes are distributed uniformly in this direction leading to $z^{+} \approx 16$ and a total of 7.8 million nodes. The second grid (LES-W), has a spanwise extent of $L_{z}=0.2 c$, discretized by 400 nodes such that $z^{+}$remains constant between the two domains for direct comparison giving a total of 39 million nodes. It is feasible to compute the narrow span (LES-N) on moderate-sized computers, while the large span (LESW) currently requires large-scale high-performance machines. Previous works on turbine blades have found spanwise extents of $0.10 c$ [31], $0.15 c$ [32], and $0.20 c[33]$ to be sufficient. Definitions of the global coordinate system and the inflow angle, $\beta$, are shown in Fig. 1 along with a cut through the computational mesh, which has every other grid line shown for clarity. The global velocity variables $u, v$, and $w$ are defined in the axial, transverse, and spanwise directions, respectively. The flow inlet angle is described as positive when it is above the design angle of the blade, and negative below the design angle.

Inlet total pressure and total temperature were set to match the experimental inlet Mach number.

Table 1: Experimental test case parameters

\begin{tabular}{llll}
\hline Case & $\beta$ & $M_{1}$ & $R e$ \\
\hline 1 & 24.49 & 0.2206 & 690000 \\
2 & 28.00 & 0.2151 & 648000 \\
3 & 32.95 & 0.2079 & 630000 \\
4 & 37.07 & 0.2205 & 683000 \\
5 & 38.91 & 0.2164 & 676000 \\
6 & 42.90 & 0.2221 & 686000 \\
7 & 45.96 & 0.2224 & 690000 \\
8 & 40.00 & 0.2500 & 740000 \\
9 & 43.40 & 0.2724 & 774000 \\
\hline
\end{tabular}




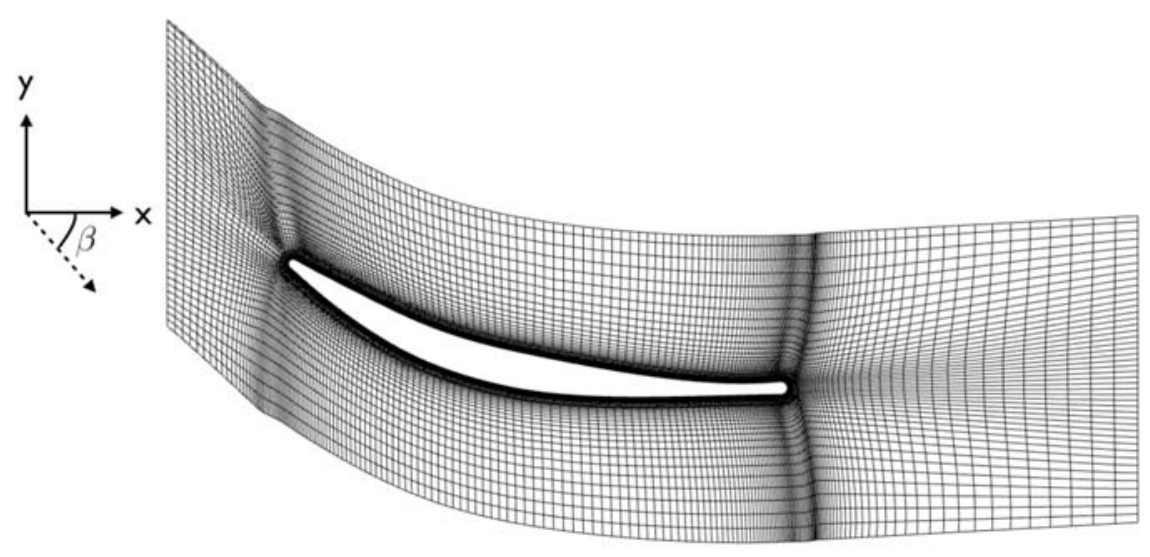

Fig. 1 Blade geometry and grid. Every second grid line shown for clarity

The experimental cascade did not feature an upstream transient wake passage and the background turbulence level remained constant at roughly 1.4 per cent.

Random velocity fluctuations with a Gaussian distribution are added at the inlet plane. These fluctuations are uncorrelated in space and time, and due to the sawtooth like distribution are rapidly damped by the numerical smoothing and the SGS model as they convect towards the blade. The inlet fluctuations were set at about 20 per cent of the freestream velocity so that at the leading edge the intensity had reduced down to around 1 percent, similar to the experimental value. While the simulation has a measurable fluctuation at the leading edge, since the grid is relatively coarse upstream of the blade and techniques such as recycling have not been employed, the simulation fluctuations are not realistic turbulence. However, they do seem to provide an appropriate seed for the growth of disturbances in the early development of the boundary layer.

A subsonic pressure outflow condition is applied at the outlet plane. The upper and lower boundaries of the domain are periodic. Due to boundary layer growth in the experimental cascade, the axial velocity density ratio (AVDR) is 1.025; hence, periodic boundary conditions cannot be used in the spanwise direction and instead these are defined as inviscid walls. While approximating the flow by assuming an AVDR of unity would allow the replacement of spanwise inviscid walls with a second pair of periodic boundaries, the unstructured industrial code used in this study is only capable of handling a single pair of periodic boundaries, and so this option was unable to be tested. The AVDR is set to match that found in the experimental case $8, \beta=40^{\circ}$.

In order to avoid the high computational cost involved in propagating starting transient flow features through the domain, the initial LES flow field in each case is obtained from a partially converged RANS solution. This RANS solution was also used to evaluate a local convective time scale in each finite volume cell based upon the cube root of the volume divided by the local velocity magnitude. The computational time step was then set to be one quarter of the smallest convective time scale present. This value was $5.8 \times 10^{-5} \mathrm{c} / V_{\text {ref }}$ (where $V_{\text {ref }}$ is the inlet velocity for case 8). The Smagorinsky constant is set to $C_{s}=0.1$. Statistical samples were taken every ten time steps over a period of 300000 time step increments. On the LES-N grid, the simulations required approximately 24 wall hours on 512 processor cores, while the LES-W simulations needed $48 \mathrm{~h}$ when run on 1024 processor cores which is consistent with the ratio of the sizes of the computational grids. A total of 18 calculations have been carried out.

\section{RESULTS}

To be consistent with the presentation of the experimental data, all velocity data are normalized by the inlet velocity, $V_{i n}$, of the simulation. Flow statistics of the blade are obtained at mid-span and presented in a co-ordinate system that is local to the blade surface, with $u_{t}$ defined as the velocity tangential to the blade. Instantaneous flow visualizations are presented in the global co-ordinate system with corresponding global velocity variables. The surface pressure coefficient, $C_{p}$, is defined as

$$
C_{p}=\frac{p-p_{0}}{\frac{1}{2} \rho V_{i n}^{2}}
$$

where $p_{0}$ is the inlet static pressure. Results from the simulations of Chen et al. [18], using the linear lowReynolds number variant of the Launder and Sharma $k-\varepsilon$ model [34] will be plotted alongside the current simulation data where available. 


\subsection{Flow structure}

Large eddy simulation offers an advantage over traditional RANS methods in that its time-dependent nature allows the structure of the turbulent flow to be understood through visualization techniques. At negative incidence, it is likely that the pressure side boundary layer will undergo a laminar separation and transition to turbulence, with similar behaviour occurring on the suction-surface when the incidence angle is positive.

\subsubsection{Positive incidence}

The global axial velocity distribution at a distance of $r / c=8.95 \times 10^{-4}$ normal to the suction surface in case 7 is shown in Fig. 2 for both grids. Both flow-fields share several features: the flow separates near the leading edge, characterized by the region of reverse flow between $0.0<x / c_{t}<0.08$. The flow then reattaches to the blade surface and is turbulent in nature. The reattachment of the flow prompts the appearance of axially-orientated streaks in the flow, which indicate the presence of contra-rotating vortex pairs within the turbulent boundary layer on the convex surface. These streaks persist along the remainder of the blade surface until the flow separates from the trailing edge. The nature of these streaks is similar to those found in other numerical studies of separation bubbles formed on flat plates $[35,36]$. While the two images are qualitatively similar, it is evident that only a very small number of these streaks are apparent in the LES-N grid simulation (Fig. 2(b)), suggesting that the narrow domain is restricting the development of the boundary layer. In comparison, the flow field from the LES-W grid in Fig. 2(a) shows several streaks across the span of the blade.

Cuts through the suction-side boundary layer at an axial location of $x / c_{t}=0.82$ are shown in Fig. 3 for both grids. In the LES-W simulation, the boundary layer is dominated by the presence of 'mushroomshaped' eruptions. The stems of these structures correspond to the spanwise locations, where the low-speed streaks are apparent in Fig. 2(a). Several scales of structure are also apparent, with small structures residing near the surface of the blade, and progressively larger structures dominating the majority of the boundary layer thickness. These flow patterns are indicative of contra-rotating vortex pairs in the boundary layer and have widely been observed in both experimental and numerical investigations of flat-plate boundary layers [35, 37]. In the LES-N simulation, however, such flow structure is not immediately apparent. The large structures evident in the boundary layer of LES-W simulation have a spanwise scale of $0.05 c$, greater than the domain extent of the LES-N simulations. It is therefore reasonable to conclude that the development of the boundary layer is adversely affected by the narrow span of the LES-N simulations when the boundary layer thickness approaches $\delta=0.04 c$.

\subsubsection{Negative incidence}

Figure 4 shows the global axial velocity distribution at a distance of $r / c=8.95 \times 10^{-4}$ normal to the pressure surface for case 2 . As the flow is at high negative incidence, there is a laminar separation bubble near to the leading edge of the pressure surface. The flow evolution on the pressure surface in the simulation is similar to that described above for case 7 . The LES-W

(a)

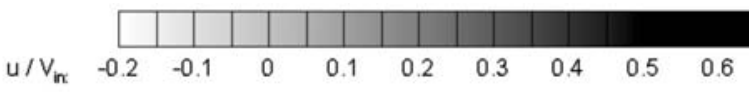

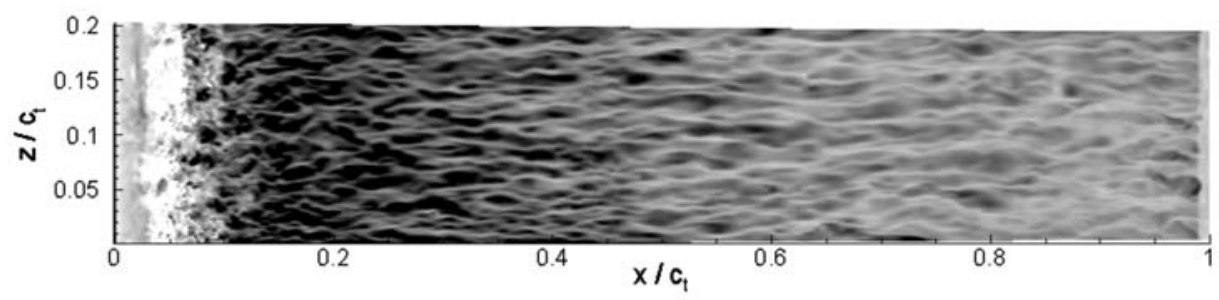

(b)

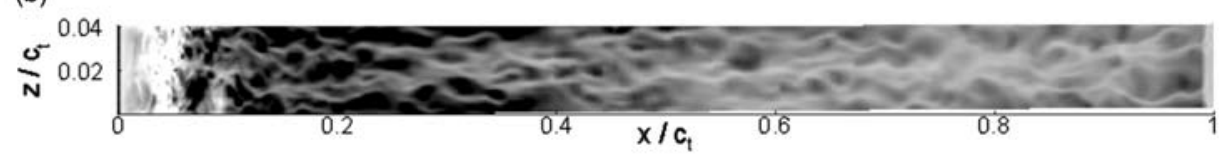

Fig. 2 Global axial velocity at a distance of $r / c=8.95 \times 10^{-4}$ normal to suction surface in case 7, (a) LES-W, (b) LES-N 

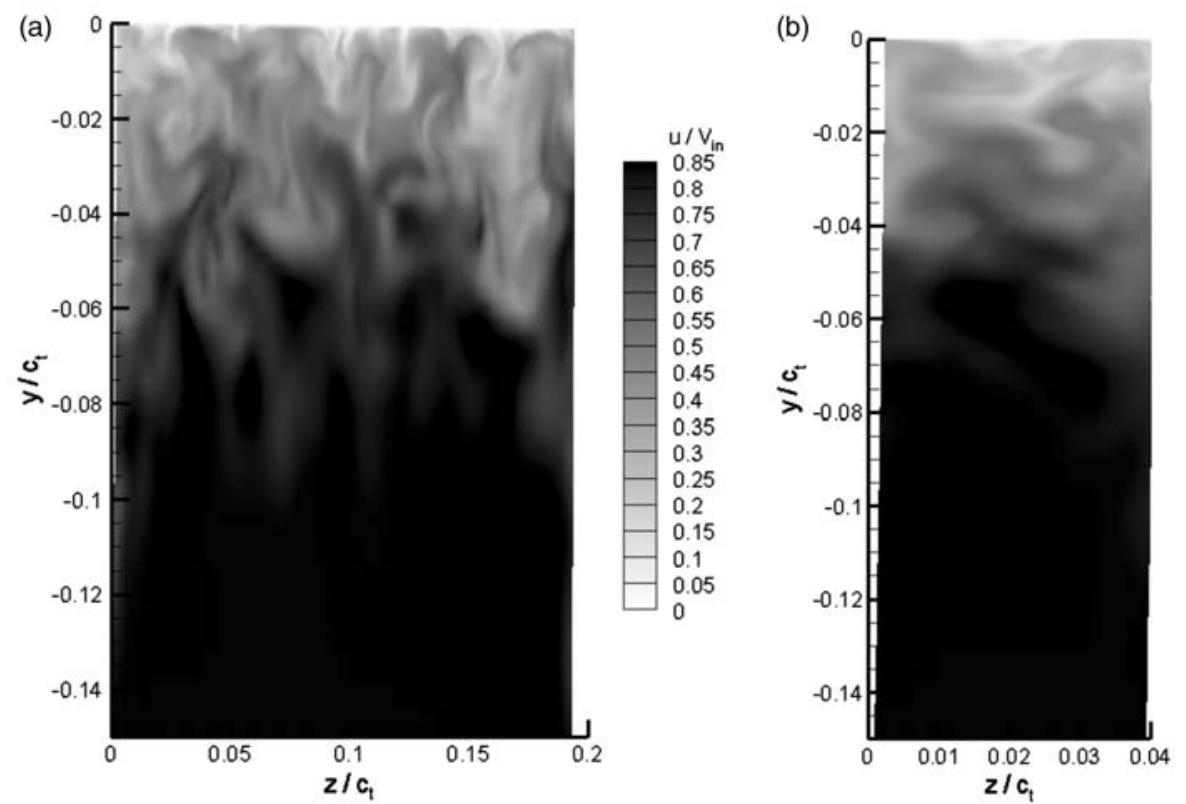

Fig. 3 Axial velocity distribution in suction-side boundary layer at $x / c_{t}=0.82$ in case 7, (a) LES-W, (b) LES-N
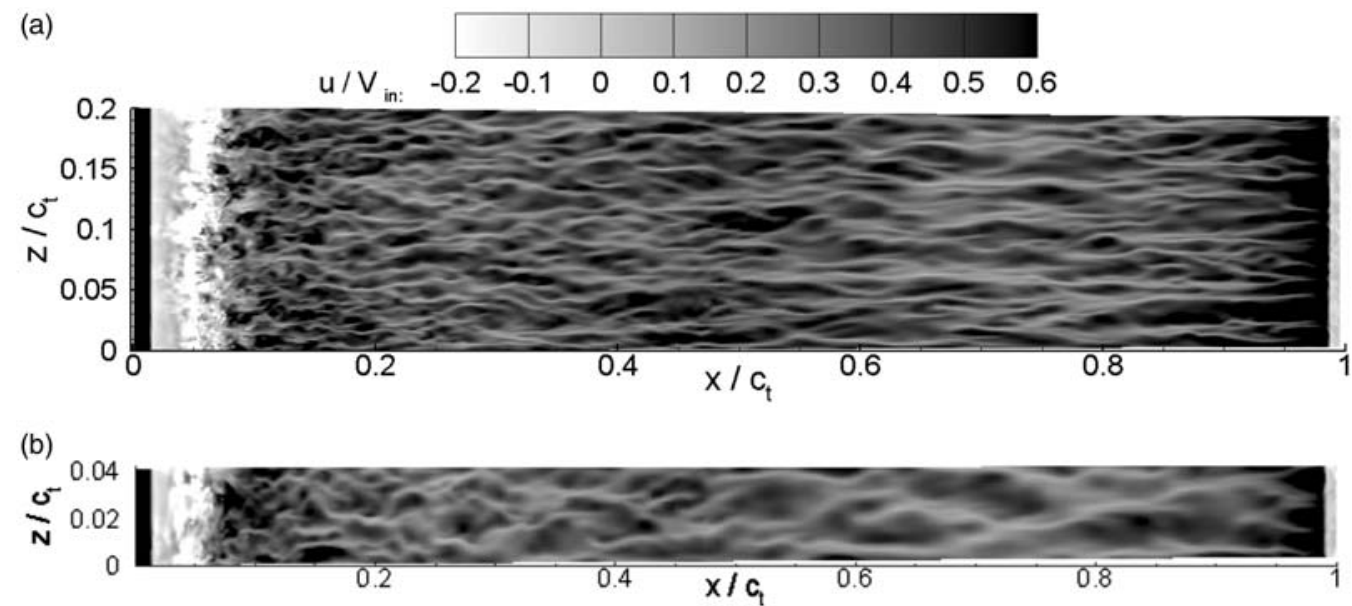

Fig. 4 Global axial velocity at $r / c=8.95 \times 10^{-4}$ normal to pressure surface in case 2, (a) LES-W, (b) LES-N

simulation in Fig. 4(a) displays several rows of streaks across the span of the blade, while the LES-N simulation in Fig. 4(b) displays very few streaks, suggesting that at high negative incidence, the flow is also being confined by the narrow domain.

The instantaneous vortex structure in the region near the leading edge of the case 2 simulations is shown in Fig. 5, with the $Q-$ criterion [38] used to highlight the vortex cores. On both grids, the laminar separation on the pressure surface produces coherent spanwise-orientated $\mathrm{K}-\mathrm{H}$ vortices that are shed by the shear layer on top of the bubble. These laminar $\mathrm{K}-\mathrm{H}$ rollers are inviscidly unstable and eventually undergo a transition to turbulence, and reattach as a turbulent boundary layer. In Fig. 5(a) the $\mathrm{K}-\mathrm{H}$ rollers are deformed by the appearance of secondary, axially orientated structures that appear in the braid region between primary rollers. The presence of this axial vorticity breaks down the spanwise-orientated $\mathrm{K}-\mathrm{H}$ structures and results in the appearance of hairpin vortices in the re-attaching boundary layer, found in other numerical studies of separating boundary layers $[39,40]$. The flow from case LES-N, shown in Fig. 5(b), does not show any significant evidence of axially-orientated vorticity in the region between primary rollers. 

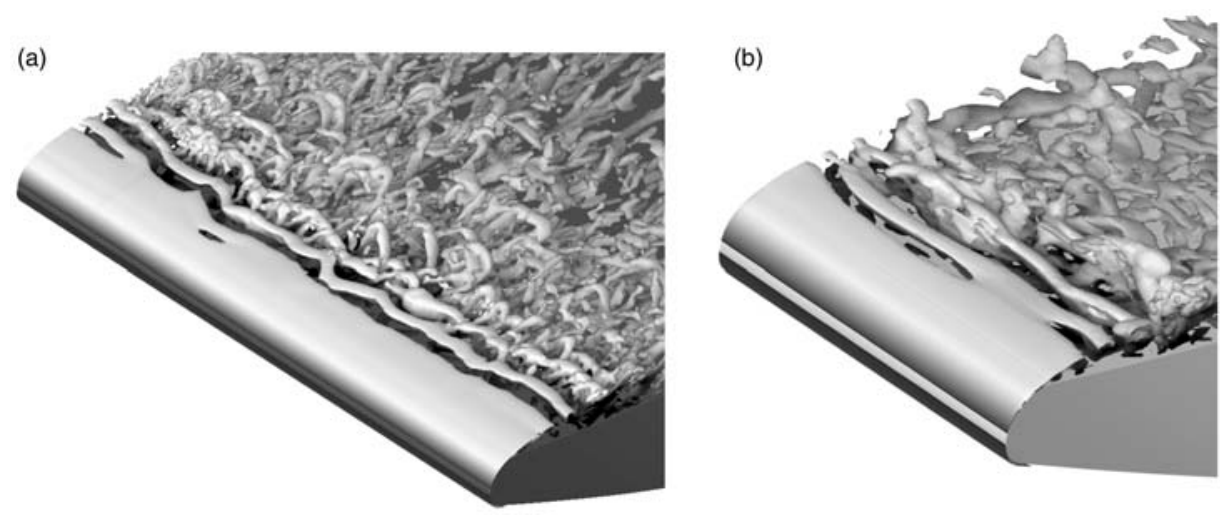

Fig. $5 Q>0$ iso-surface of case 2, (a) LES-W, (b) LES-N simulation

The data presented above show that the narrow spanwise domain imposed in the LES-N simulations has a significant impact on the evolution of the flow around the blade. From these observations of the flow structure, it would be expected that the narrow span simulation would give poor mean flow and loss predictions. This is investigated in the following section.

\subsection{Flow statistics}

Surface pressure distributions at mid-span for cases 1 and 6-8 are shown in Fig. 6 along with comparisons from the RANS solutions of Chen et al. [18] where available. The agreement between simulations on both LES grids and the experimental data is very favourable. Despite some fundamental differences in the unsteady flow structure for the narrow and wide domain, this has little effect on the pressure distributions. The LES does appear to over-predict the effect of the laminar separation bubble on the suction surface in case 8 , while no separation bubble is evident in the RANS data of Chen et al.

Boundary layer profiles recorded at mid-span from three locations on the suction side of the blade are shown in Fig. 7 for case $7, \beta=46^{\circ}$. At this high positive incidence angle, the flow has a laminar separation bubble near to the leading edge of the suction surface. The laser doppler velocimetry technique used in the experiment was unable to determine velocity data in the separation bubble, hence the lack of data points near the wall at $x / c=0.052$. Traditional RANS methods fail to capture the separation, while the LES-W simulation successfully predicts reverse flow at this location. In the LES-N simulation, the flow has reattached at this particular station. At this high angle off-design case, the boundary layers are thicker with an experimental displacement thickness of about $0.04 c$ at the trailing edge. Since the chordwise span is only $0.04 c$ then some structures in the boundary layer will likely span the domain and so be influenced by the inviscid side walls. If the side walls were replaced by a second periodic boundary, then the structures would be coupled across the boundary so also giving an incorrect flow behaviour with overly strong spanwise structures.

It is evident that LES-W produces the closest prediction to the experimental boundary layer data, with the LES-N data predicting a boundary layer that is somewhat smaller than the experimental data. Interestingly, the boundary layer RANS data of Chen et al. produces similar predictions to the LES-N simulation. The boundary layer profiles can be used to obtain the edge velocity of the flow, defined as the maximum velocity in the boundary tangential to the blade surface. In order to determine the location of the edge of the boundary layer, an approach based on that of Stock and Haase [41] is used. This estimates the location of the edge of the boundary layer from the location of the local maximum in moment of velocity gradient

$$
\delta=\left.1.936 y\right|_{(y|\mathrm{~d} u / \mathrm{d} y|)_{\max }}
$$

The edge velocity distribution at mid-span from the LES of case 7 is shown in Fig. 8. As with the surface pressure distributions in Fig. 6 , the edge velocity is well predicted on both wide and narrow grids.

Computed boundary layer parameters are shown in Fig. 9 for case 7. The boundary layer thickness on the suction surface is well predicted on the LES-W domain, while the LES-domain shows significant under-prediction of this quantity. As the boundary layer thickness at the trailing edge was reported as $\delta=0.126 c$ in the experiment, it is not surprising that a domain with a spanwise extent of $L_{z}=0.04 \mathrm{c}$ fails to reasonably capture the development of the boundary layer at this incidence. On the pressure surface, the boundary layer thickness is poorly predicted on both grids. The reason for this under-prediction is apparent in the shape factor distribution, shown in Fig. 9(c); 

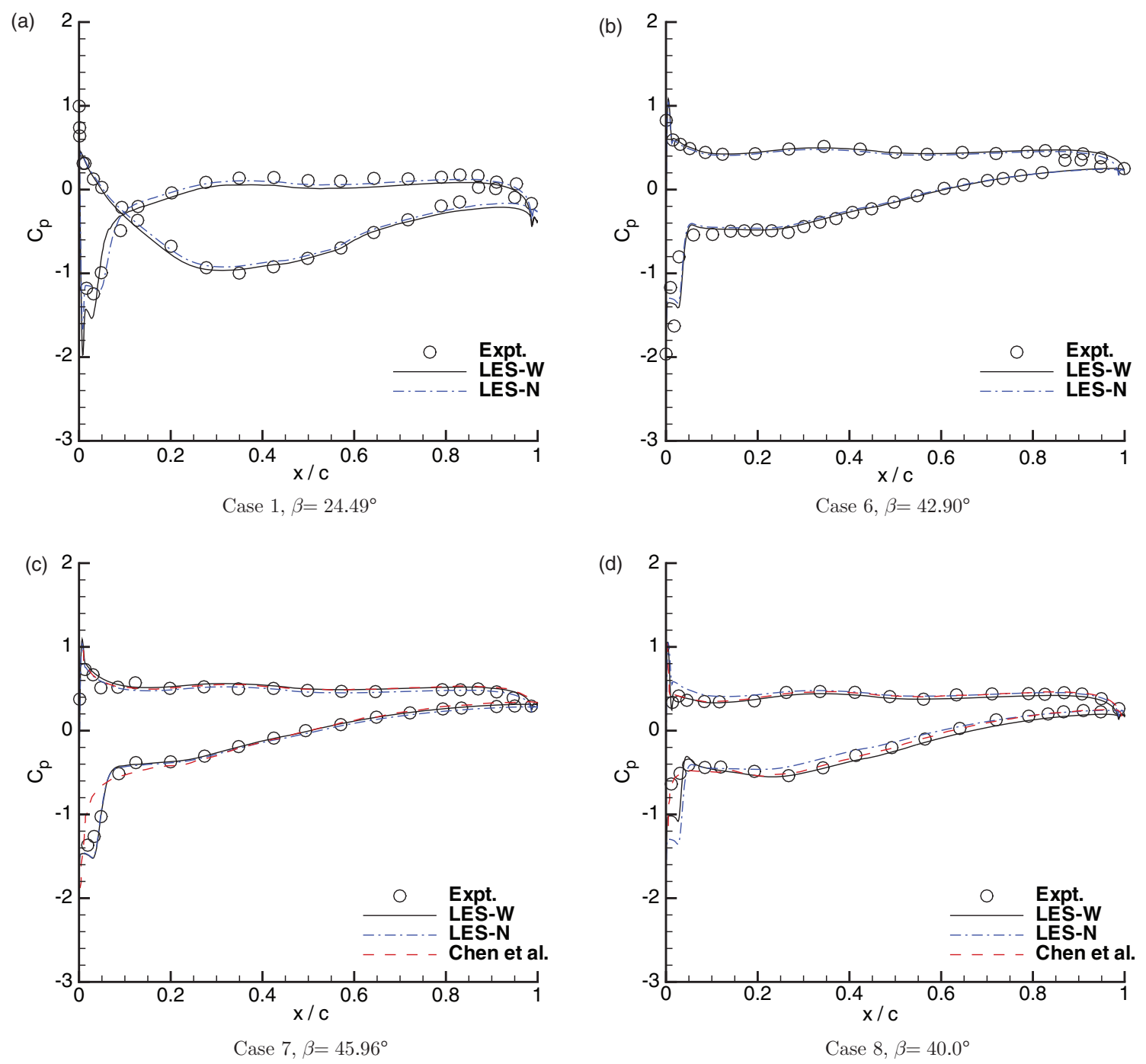

Fig. 6 Surface pressure distributions

in the experiment, the flow underwent a natural transition to turbulence at approximately 40 per cent of chord, while the LES presented here do not capture this feature and the boundary layers remain laminar up to the trailing edge of the blade. The grid used is not sufficiently well refined to resolve the instability waves that precipitate the transition to turbulence, and refinement of the grid to capture this feature would produce a mesh that approaches DNS and would therefore be computationally impractical for a flow of this Reynolds number. The RANS data of Chen et al. highlight a limitation of traditional RANS models - all the scales of motion in the flow are assumed to be turbulent and hence the boundary layer on the pressure surface is turbulent over the entire blade surface. On the suction side, the shape factor distributions computed from both LES presented agree well with the experimental data.

The displacement thickness of the suction-side boundary layer computed from the LES does not agree well with the experimental data. However the data of Chen et al. and other numerical studies of the cascade $[14,17]$ show similar trends, with no published numerical study producing data that match the experimental values.

It is apparent that, at the off-design inflow angle of case 7 at least, the LES-W simulation offers some improvement over 'thin-slice' LES and RANS simulation methods. Boundary layer properties from case 8 , $\beta=40.0^{\circ}$, nearer to the design angle of the blade are shown in Fig. 10. The suction-side boundary layer thickness of the LES-W simulation is in extremely good agreement with the experimental data, while the LES-N computation again shows some underprediction of the quantity. As noted for case 7, the failure of the grid to capture the natural transition on the pressure surface at positive incidence results in a significantly thinner pressure-side boundary layer than recorded by the experiment. The displacement thickness of the suction-side boundary layer at 


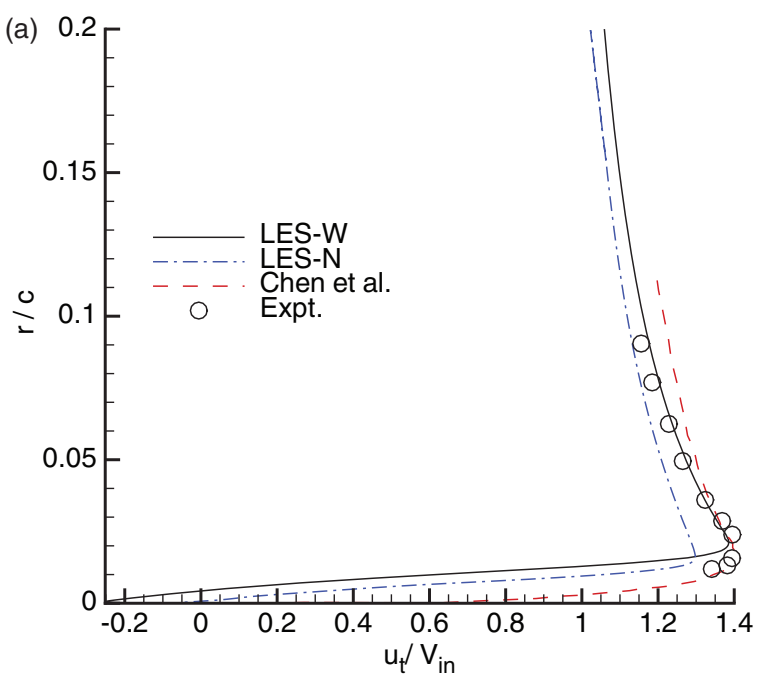

$\mathrm{x} / \mathrm{c}=0.052$

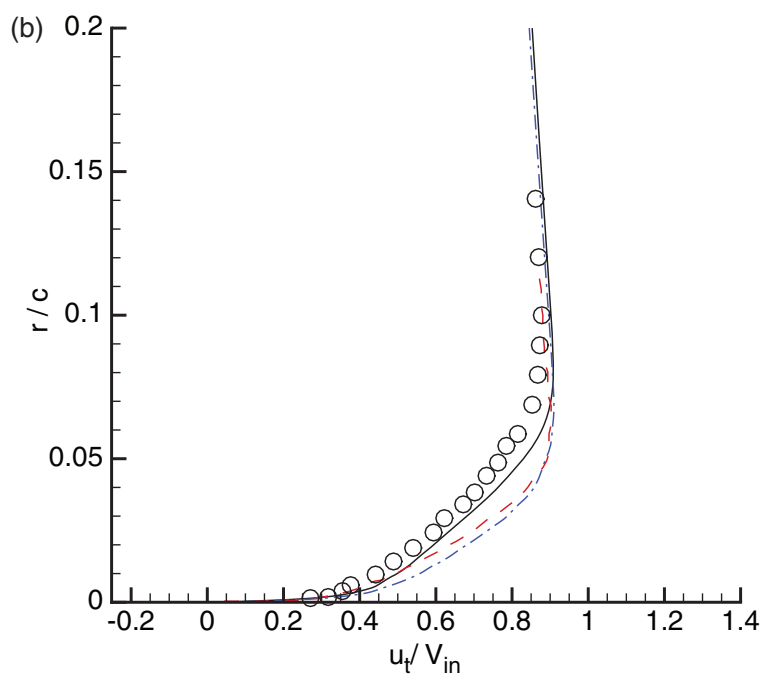

$\mathrm{x} / \mathrm{c}=0.643$

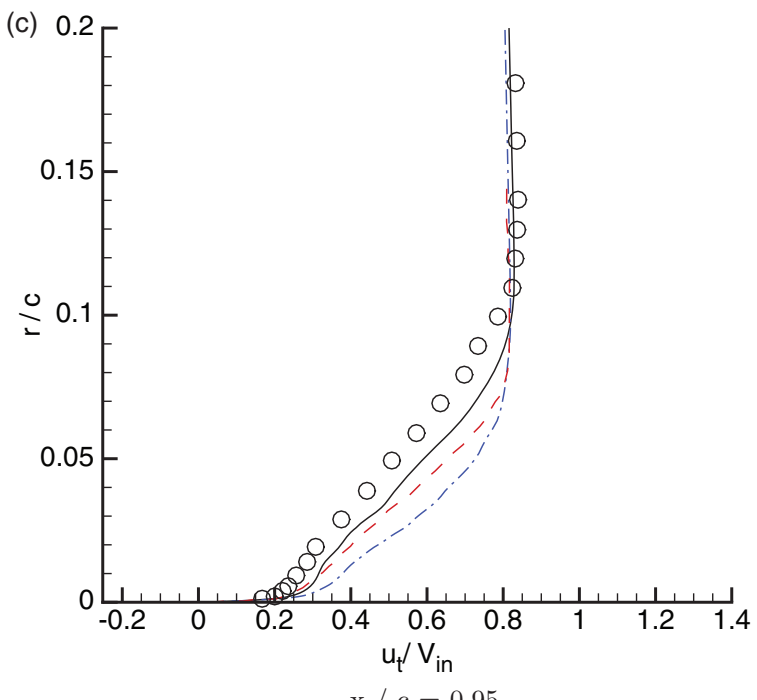

Fig. 7 Boundary layer velocity profiles for case 7

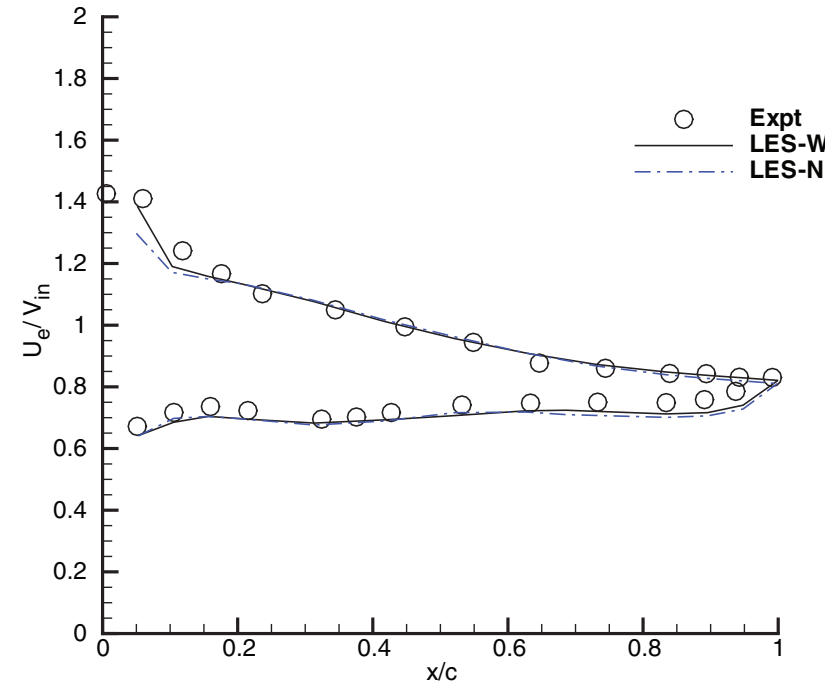

Fig. 8 Edge velocity distribution in case 7 the trailing edge is again under-predicted by both LES and RANS methods, although the RANS data is slightly closer to the experimental values near the trailing edge. The shape factor distribution of the suction-side boundary layer is not replicated well by the simulations presented here, while the pressure surface shape factor confirms the laminar nature of the LES boundary layers, and the turbulent nature of the RANS boundary layer.

\subsection{Loss estimation}

The loss in the simulations is computed using the equation

$$
\omega=\frac{P_{T 0}-P_{T 2}}{P_{D 1}}
$$



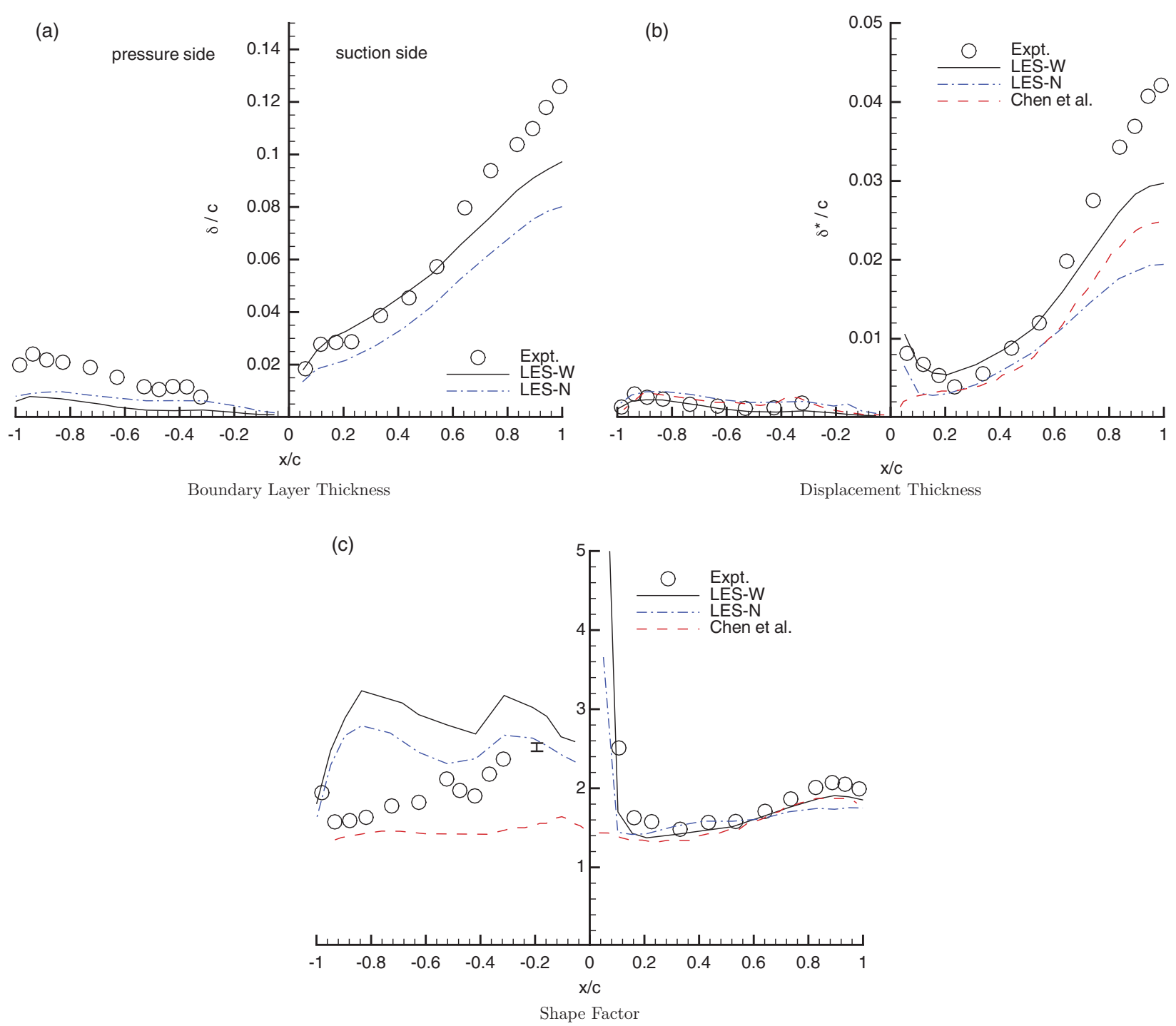

Fig. 9 Boundary layer properties for case 7

where $P_{T 0}$ and $P_{T 2}$ are the averaged total pressures at the inlet and exit planes, respectively, and $P_{D 1}$ is the averaged dynamic pressure at the inlet. In all simulations, $P_{T 2}$ is obtained at a plane $0.01 \mathrm{c}$ upstream of the exit boundary, in order to avoid any potential contamination from the exit boundary condition.

The variation of the loss coefficient across the span of the domain from case 9 is shown in Fig. 11 for both computational domains. In both simulation types, the loss increases dramatically near to the spanwise boundaries, an effect caused by the imposition of inviscid wall conditions. The structure in the turbulent flow impinges on the inviscid spanwise boundaries and is displaced vertically along it, producing an artificially enhanced boundary layer thickness near the spanwise boundaries. A representative plot of the spanwise variation of the boundary layer thickness, $\delta$, across the blade span at the trailing edge in case 9 is shown in Fig. 12. The boundary layer thickness in the LES-W simulation remains reasonably constant over the middle 70 percent of the span, while in the LES-N simulation, the boundary layer can only be considered free from the influence of the wall in a region of \pm 5 per cent of mid-span. Given that the boundary layer thickness at this location, $\delta=0.07 c$, is larger than the span of the domain, it is also reasonable to conclude that the development of the boundary layer, and consequently the wake, will be affected by the narrow span.

Producing a reliable estimation of the loss from the LES-N simulations is therefore difficult, and the loss is computed from these simulations, by taking single-point data obtained at mid-span. In the LES-W simulations, there is a substantial proportion of the flow that in unaffected by the inviscid walls; hence, the loss estimation is obtained with 15 percent of the span near each inviscid boundary ignored. The computed loss coefficient 


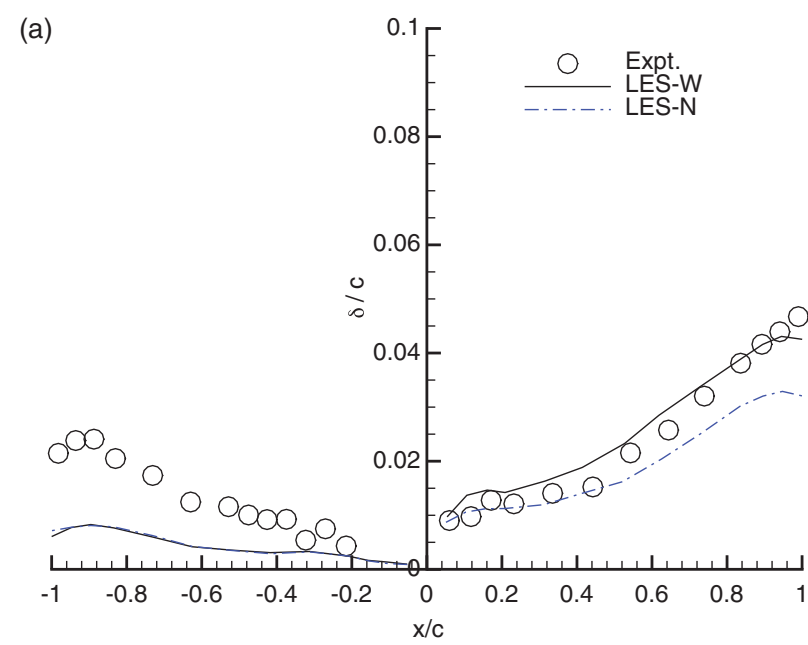

Boundary Layer Thickness

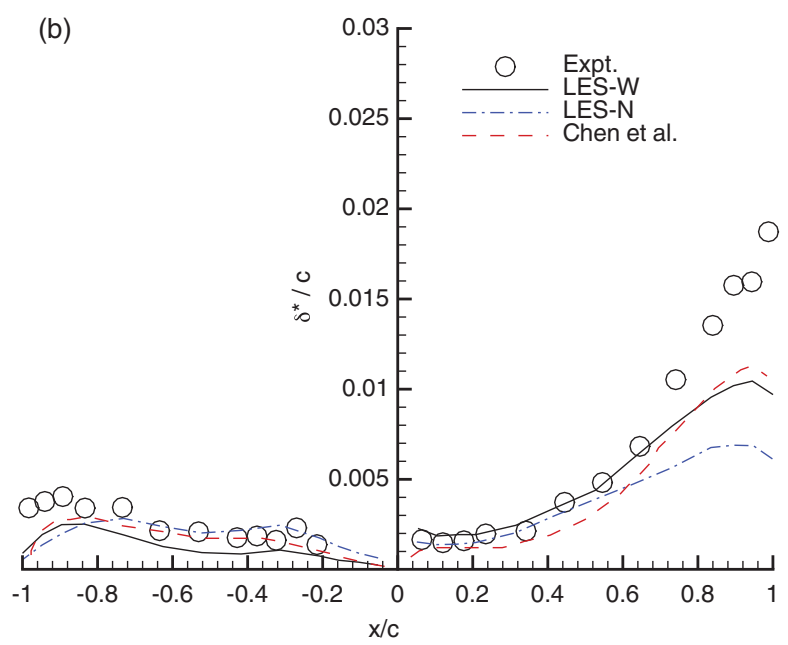

Displacement Thickness

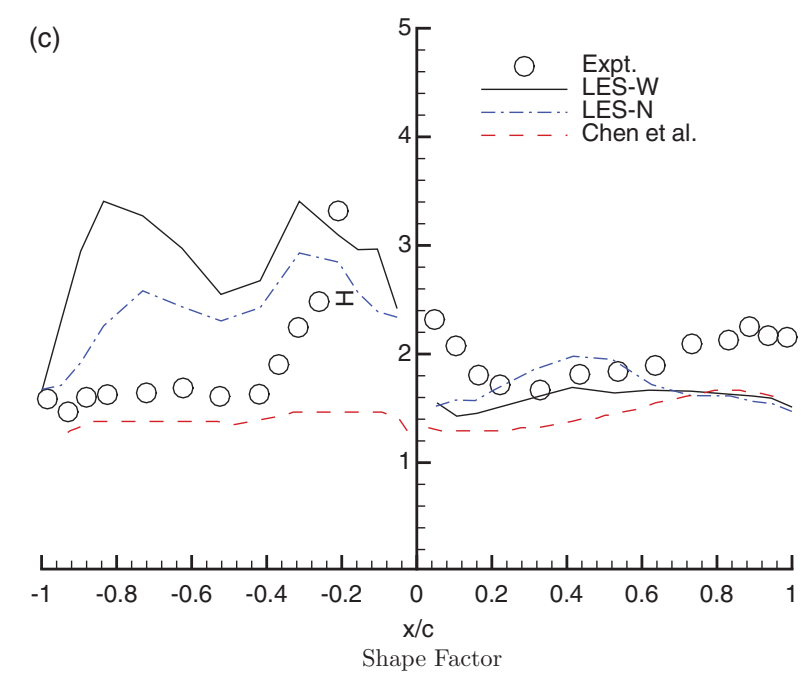

Fig. 10 Boundary layer properties for case 8

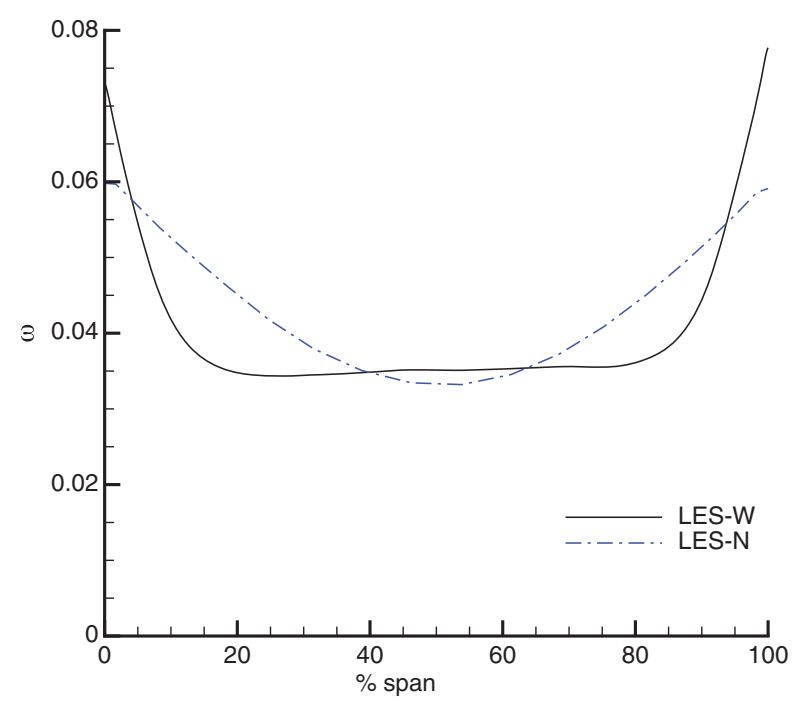

Fig. 11 Loss variation across the span of the domain in case 9 for the simulations are shown in Fig. 13, along with the experimental data and those obtained by Chen et al. Near the design angle, the LES-W simulations produce a reasonable estimate of the loss, while at high negative incidence the loss estimate is too large. At high positive incidence, the computed loss from the LES-W grid is too low when compared to the experiment, a trend that is common with other numerical studies of the cascade $[14,17]$. While the trend of the loss estimation from the LES-N simulations may appear to be in good agreement with experiment, it should be emphasized that the flow in these simulations is compromised by both confinement of the narrow span and the parasitic effects of the inviscid spanwise boundaries, which artificially thickens the boundary layer and wake flow. The LES-N simulations should therefore be considered as an unreliable method to estimate the loss in the cascade. 


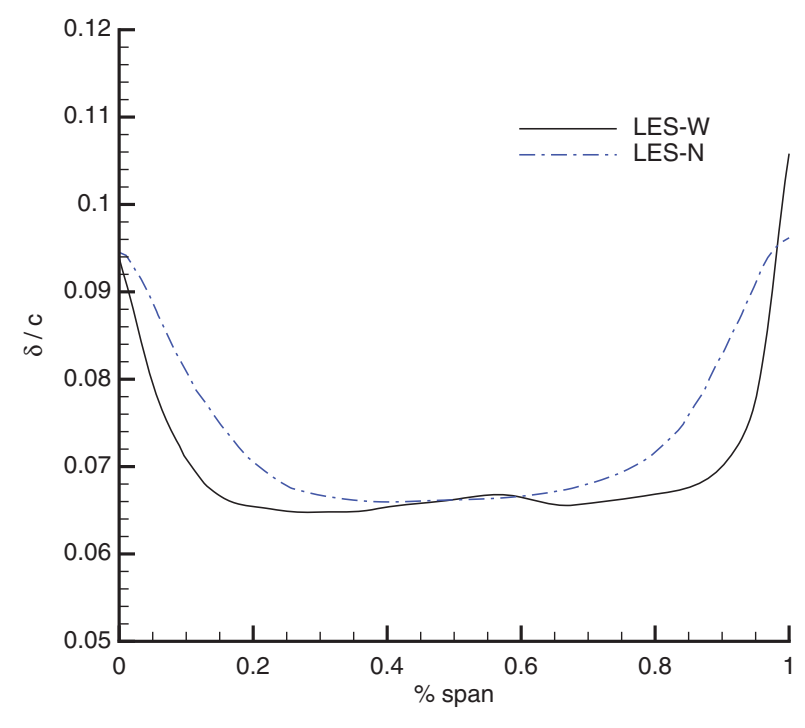

Fig. 12 Suction-side boundary layer thickness variation across the span of the domain in case 9

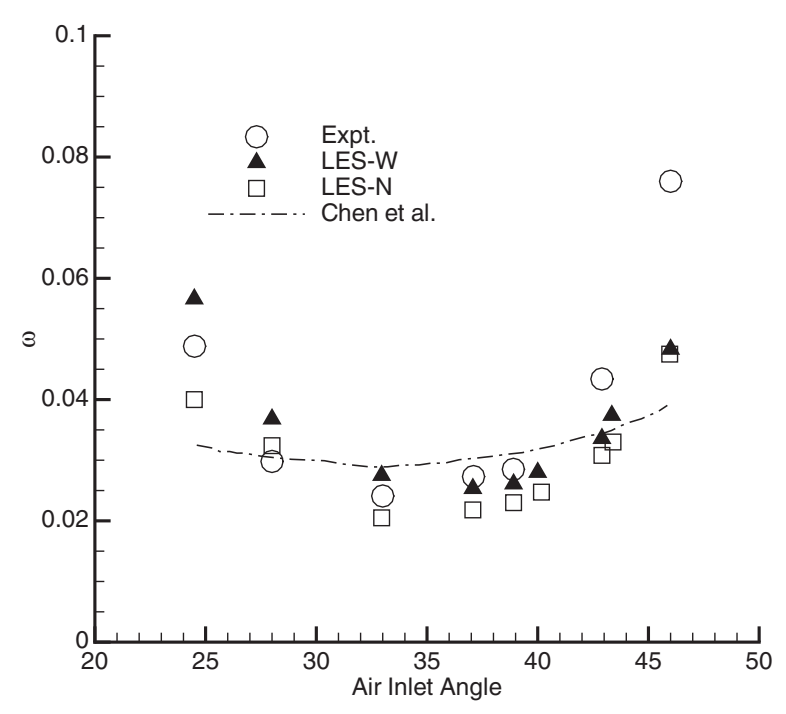

Figure 13 Loss estimation from the simulations

\section{CONCLUSIONS}

Results have been shown for LES of a compressor cascade over a wide range of inlet angles on two grids that model a $0.04 c$ span and a $0.20 c$ span. While the surface pressure distribution of the flow and boundary layer development is well predicted over the range of inlet angles on both grids, the narrow span of the thin-slice simulations confines the flow and restricts its overall development when the boundary layer thickness approaches the spanwise domain extent. As the AVDR of the flow enforces the use of inviscid spanwise boundaries, the interaction of the turbulence structure and the inviscid wall results in an artificial thickening of the boundary layer, which prevents a reliable estimate of the loss from thin-slice LES to be obtained. While the wide domain simulations also suffer from the parasitic influence of the inviscid boundaries, the spanwise extent is sufficient for a reasonable estimate of the loss from the simulation to be obtained. The flow statistics obtained from the wide domain simulations are in reasonable agreement with experiment, and offer some improvement of loss prediction over traditional RANS methods at off-design angles. The time-dependent nature of the LES allows the turbulence structure of the flow to be visualized, and the mechanisms of flow development to be understood.

The aim of this study is to assess the capability of LES to be used in an industrial context - if limits of computational resources means that the spanwise domain is too small, then LES is a poor tool to predict losses in compressor cascades. Ultimately, LES will be most useful if resources are available to compute the complete 3D blade including tip flow effects and this is the current focus of further work in this area.

\section{FUNDING}

This work was funded by the UK Technology Strategy Board and Rolls-Royce plc under the CFMS Core Programme (TP/L3001H). The simulations were performed on HECToR, the UK National Supercomputing Facility, funded under the UKAAC-2 Framework, EPSRC Grant No. EP/F005954/1.

\section{ACKNOWLEDGEMENTS}

The authors would like to thank Leigh Lapworth of Rolls-Royce plc for assistance in computing the loss of the simulations.

(C) Authors 2011

\section{REFERENCES}

1 Wissink, J. G. and Rodi, W. Direct numerical simulation of flow and heat transfer in a turbine cascade with incoming wakes. J. Fluid Mech., 2006, 569, 209-247.

2 Michelassi, V., Wissink, J. G., Frohlich, J., and Rodi, W. Large-eddy simulation of flow around low-pressure turbine blade with incoming wakes. AIAA J., 2003, 41, 2143-2156.

3 Wu, X. and Durbin, P. A. Evidence of longitudinal vortices evolved from distorted wakes in a turbine passage. J. Fluid Mech., 2001, 446, 199-228.

4 Wissink, J. G., Rodi, W., and Hodson, H. P. The influence of disturbances carried by periodically incoming wakes on the separating flow around a turbine blade. Int. J. Heat Fluid Flow, 2006, 27, 721-729. 
5 Matsuura, K. and Kato, C. Large eddy simulation of compressible transitional cascade flows. J. Fluid Sci. Tech., 2007, 2, 558-569.

6 You, D., Mittal, R., Wang, M., and Moin, P. Computational methodology for large-eddy simulation of tip-clearance flows. AIAAJ., 2004, 42, 271-279.

7 You, D., Mittal, R., Moin, P., and Wang, M. Effects of tip-gap size on the tip-leakage flow in a turbomachinery cascade. Phys. Fluids, 2004, 18, 105102.1105102.14 .

8 You, D., Wang, M., Moin, P., and Mittal, R. Largeeddy simulation analysis of mechanisms for viscous losses in a turbomachinery tip-clearance flow. J. Fluid Mech., 2007, 586, 177-204.

9 Borello, D., Hankalic, K., and Rispoli, F. Computation of tip-leakage flow in a linear compressor cascade with a second-moment turbulence closure. Int. J. Heat Fluid Flow, 2007, 28, 587-601.

10 Zaki, T. A., Wissink, J. G., Durbin, P. A., and Rodi, W. Direct computations of boundary layers distorted by migrating wakes in a linear compressor cascade. Flow, Turb. Combust., 2009, 83, 307-322. (DOI 10.1007/s10494-009-9216-0.

11 Sanger, N. L. and Shreeve, R. P. Comparison of calculated and experimental cascade performance for controlled-diffusion compressor stator blading. J. Turbomach., 1986, 108, 42-50.

12 Elazar, Y. and Shreeve, R. P. Viscous flow in a controlled diffusion compressor cascade with increasing incidence. J. Turbomach., 1990, 112, 257-265.

13 Shreeve, R. P., Elazar, Y., Dreon, J. W., and Baydar, A. Wake measurements and loss evaluation in a controlled diffusion compressor cascade. J. Turbomach., 1991, 113, 591-599.

14 Elazar, Y. A mapping of the viscous flow in a controlled diffusion cascade using laser Doppler velocimetry and preliminary evaluation of codes for the prediction of stall. Ph.D. Thesis, 1988 (Naval Postgraduate School, Monterey, California).

15 Dreon, J. W. Controlled diffusion compressor blade wake measurements. MS Thesis, 1986 (Naval Postgraduate School, Monterey, California).

16 Koyuncu, Y. Report of tests of a compressor configuration of CD blading. M.S. Thesis, 1984 (Naval Postgraduate School, Monterey, California).

17 Ho, Y. K., Walker, G. J., and Stow, P. Boundary layer and Navier-Stokes analysis of a NASA controlled-diffusion compressor blade, 1990, ASME Paper 90-GT236, Brussels, Belgium.

18 Chen, W. L., Lien, F. S., and Leschziner, M. A. Computational prediction of flow around highly loaded compressor-cascade blades with non-linear eddy-viscosity models. Int. J. Heat Fluid Flow, 1998, 19, 307-319.

19 McMullan, W. A. and Page, G. J. Large eddy simulation of a controlled-diffusion cascade blade at varying flow inlet angles, 2009, ASME Paper GT2009-59668, Orlando, USA.

20 McMullan, W. A. and Page, G. J. Large eddy simulation of a controlled-diffusion cascade. Flow Turbul. Combust., 2011, 86(2), 207-230. DOI: 10.1007/ s10494-010-9314-Z.
21 Crumpton, P. I., Moinier, P., and Giles, M. G. An unstructured algorithm for high Reynolds number flows on highly stretched grids. In Tenth International Conference on Numerical Methods for Laminar and Turbulent Flow, 1997.

22 Tristanto, I. H., Li, Q., Page, G. J., and McGuirk, J. J. On the effect of convective flux formation for LES of compressible flows using hybrid unstructured meshes. In AIAA Paper 2006-54940, 36TH AIAA Fluid Dynamics Conference, San Francisco, California, 5-8 June 2006.

23 Li, Q., Page, G. J., and McGuirk, J. J. Large-eddy simulation of twin impinging jets in crossflow. Aeronaut. J., 2007, 111, 195-205.

24 Page, G. J. and McGuirk, J. J. Large eddy simulation of a complete Harrier aircraft in ground effect. IPLC08-0033. In Proceedings of the International Powered lift conference, London, 22-24 July 2008.

25 Moinier, P. Algorithm developments for an unstructured viscous flow solver. $\mathrm{PhD}$ Thesis, 1998 (University of Oxford).

26 Ciardi, M., Sagaut, P., Klein, M., and Dawes, W. N. A dynamic finite volume scheme for large-eddy simulation on unstructured grids. J. Comput. Phy., 2005, 210, 632-655.

27 Ducros, F., Ferrand, V., Nicoud, F., Weber, C., Darracq, D., Gacheriu, C., and Poinsot, T. Largeeddy simulation of the shock/turbulence interaction. J. Computational Physics, 1999, 152, 517-549.

28 Smagorinsky, J. General circulation experiments with the primitive equations. Monthly Weather Rev., 1963, 91, 99-164.

29 Burgess, D. A., Crumpton, P. I., and Giles, M. B. A parallel framework for unstructured grid solvers. Programming environments for massively parallel distributed systems, 1994, pp. 97-106 (Birkhauser).

30 Hills, N. Achieving high parallel performance for an unstructured unsteady turbomachinery code. The Aeronautical J., 2007, 111, 185-193.

31 Sarkar, S. and Voke, P. R. Large-eddy simulation of unsteady surface pressure over a low-pressure turbine blade due to interactions of passing wakes and inflexional boundary layer. J. Turbomach., 2006, 128, 221-231.

$32 \mathrm{Wu}$, X. H. and Durbin, P. A. Evidence of longitudinal vortices evolved from distorted wakes in a turbine passage. J. Fluid Mech., 2001, 446, 199-228.

33 Wissink, J. G. and Rodi, W. Direct numerical simulation of flow and heat transfer in a turbine cascade with incoming wakes. J. Fluid Mechanics, 2006, 569, 209-247.

34 Launder, B. E. and Sharma, B. I. Application of the energy-dissipation model of turbulence to the calculations of flow near a spinning disc. Int. J. Heat Mass Transfer, 1974, 1, 131-137.

35 Alam, M. and Sandham, N. Direct numerical simulation of short laminar separation bubbles with turbulent reattachment. J. Fluid Mech., 2000, 403, 223-250.

36 Yang, Z. and Voke, P. R. Large-eddy simulation of boundary-layer separation and transition at a 
change of surface curvature. J. Fluid Mech., 2001, 439, 305-333.

37 Kiya, M. and Sasaki, K. Structure of a turbulent separation bubble. J. Fluid Mech., 1983, 137, 83-113.

38 Jeong, J. and Hussain, F. On the identification of a vortex. J. Fluid Mech., 1995, 285, 69-94.

39 Yang, Z. and Abdalla, I. E. Effects of free-stream turbulence on large-scale coherent structures of separated boundary layer transition. Int. J. Numer. Methods. Fluids, 2005, 49, 331-348.

40 McAuliffe, B. R. and Yaras, I. Numerical study of instability mechanisms leading to transition in separation bubbles. J. Turbomach., 2008, 130, 021006pp1-8.

41 Stock, H. W. and Haase, W. Determination of length scales in algebraic turbulence models for NavierStokes methods. AIAA J., 1989, 27, 5-14.

\section{APPENDIX}

\section{Notations}

$\begin{array}{ll}A_{i j} & \text { flux Jacobian } \partial F / \partial Q \\ c & \text { blade chord } \\ C_{p} & \text { pressure coefficient } \\ C_{s} & \text { Smagorinsky model constant } \\ E & \text { total energy per unit volume }\end{array}$

$\begin{array}{ll}F, G & \text { flux vectors } \\ H & \text { boundary layer shape factor } \\ L & \text { pseudo-Laplacian } \\ L_{z} & \text { spanwise extent } \\ n & \text { normal vector } \\ p & \text { pressure } \\ P_{T} & \text { total pressure } \\ Q & \text { state vector } \\ S_{i j} & \text { strain rate tensor } \\ t & \text { time } \\ u, v, w & \text { Cartesian components of velocity } \\ U_{n} & \text { face normal velocity component } \\ V_{i n} & \text { inlet velocity } \\ x, y, z & \text { Cartesian components of position } \\ x^{+}, y^{+}, z^{+} & \text {distance in wall units } \\ \beta & \\ \Delta & \text { inlet flow angle } \\ \delta & \text { filter width } \\ \delta * & \text { boundary layer thickness } \\ \mu_{s g s} & \text { boundary layer displacement thickness } \\ \rho & \text { SGS viscosity } \\ \varepsilon & \text { density } \\ & \text { smoothing coefficient }\end{array}$

